\title{
Overview of the predictive value of quantitative 18 FDG PET in head and neck cancer treated with chemoradiotherapy
}

\author{
J. Castellia ${ }^{\mathrm{a}, \mathrm{b}, \mathrm{c}, *}$, B. De Bari a ${ }^{\mathrm{a}}$ A. Depeursinge ${ }^{\mathrm{d}, \mathrm{e}}$, A. Simon $^{\mathrm{b}, \mathrm{c}}$, A. Devillers $^{\mathrm{f}}$, \\ G. Roman Jimenez ${ }^{\mathrm{b}, \mathrm{c}, \mathrm{g}}$, J. Prior ${ }^{\mathrm{h}}$, M. Ozsahin ${ }^{\mathrm{a}}$, R. de Crevoisier ${ }^{\mathrm{b}, \mathrm{c}, \mathrm{i}}$, J. Bourhis ${ }^{\mathrm{a}}$ \\ a Radiotherapy Department, CHUV, Lausanne, Switzerland \\ b INSERM, U1099, Rennes, F-35000, France \\ ' Université de Rennes 1, LTSI, Rennes, F-35000, France \\ ${ }^{\mathrm{d}}$ University of Applied Sciences Western Switzerland, 3960 Sierre, Switzerland \\ e Ecole Polytechnique Fédérale de Lausanne, CH-1015 Lausanne VD, Switzerland \\ ${ }^{\mathrm{f}}$ Nuclear Medecine Department, Centre Eugene Marquis, Rennes, F-35000, France \\ ${ }^{g}$ Keosys Medical imaging, 1 Impasse Augustin Fresnel, Saint-Herblain, F-44815, France \\ h Nuclear Medecine Department, CHUV, Switzerland \\ ${ }^{\mathrm{i}}$ Radiotherapy Department, Centre Eugene Marquis, Rennes, F-35000, France
}

\section{Contents}

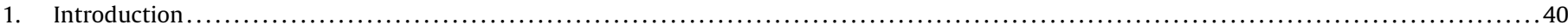

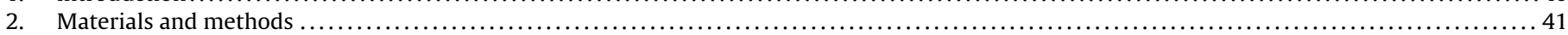

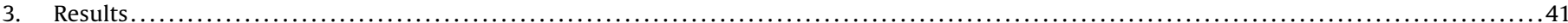

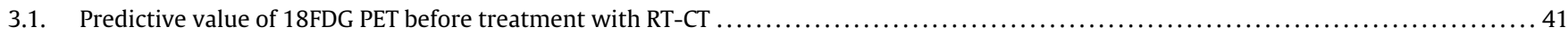

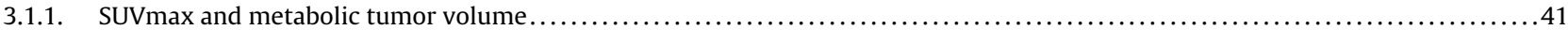

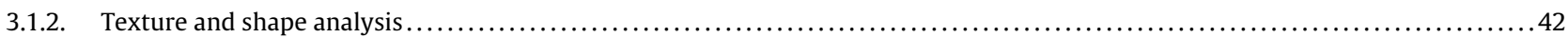

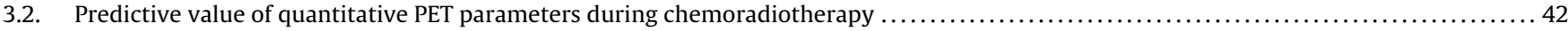

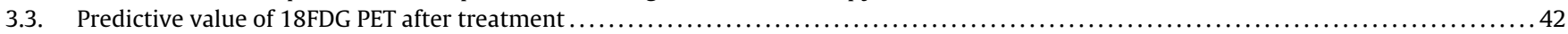

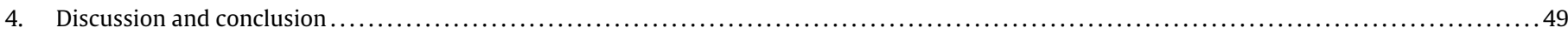

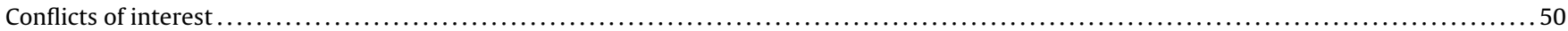

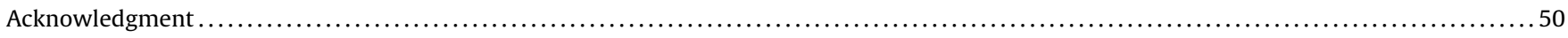

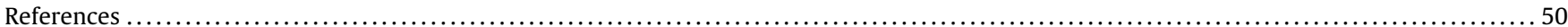

\section{A R T I C L E I N F O}

\section{Article history:}

Received 27 June 2016

Received in revised form 8 October 2016

Accepted 26 October 2016

\section{Keywords:}

PET

Head and neck cancer

Chemoradiotherapy

Clinical outcome

\begin{abstract}
A B S T R A C T
18 F-fluorodeoxyglucose (18F-FDG) positron emission tomography/computed tomography (PET/CT) allows to quantify the metabolic activity of a tumor (glycolysis) and has become a reference tool in oncology for the staging, restaging, radiotherapy planning and monitoring response in many cancers. Quantitative analyses have been introduced in order to overcome some of the limits of the visual methods, allowing an easier and more objective comparison of the inter- and intra-patients variations. The aims of this review were to report available evidences on the clinical value of quantitative PET/CT parameters in HNC.

Forty-five studies, for a total of 2928 patients, were analyzed. Most of the data available dealt with the intensity of the metabolism, calculated from the Standard Uptake Value (SUV). Metabolic Tumor Volume (MTV) was well correlated with overall survival and disease free survival, with a higher predictive value than the maximum SUV. Spatial distribution of metabolism and textural analyses seems promising.
\end{abstract}

(c) 2016 Elsevier Ireland Ltd. All rights reserved.

\footnotetext{
* Correspondence to: Department of Radiation Oncology, Centre Eugene Marquis avenue de la Bataille Flandre Dunkerque, F-35000 Rennes, France.

E-mail address: j.castelli@rennes.unicancer.fr (J. Castelli).
}

\section{Introduction}

Head and neck cancers are among the most common in the world (5th leading cancer by incidence (Parkin et al., 2005)). The American Joint Committee on Cancer (AJCC) staging is gen- 
erally used to estimate the prognosis and guide therapy (Edge and Compton, 2010). Radio-chemotherapy is a standard treatment of unresectable and/or locally advanced Head and Neck Cancers (Pignon et al., 2000; St Guily et al., 2010). Despite this treatment, the prognosis remains worst and loco-regional recurrence may occur in up to $40 \%$ patients, mostly within the first 2 -years after treatment (Chajon et al., 2013). ${ }^{18} \mathrm{~F}$-fluorodeoxyglucose ( $\left.{ }^{18} \mathrm{~F}-\mathrm{FDG}\right)$ positron emission tomography/computed tomography (PET/CT) allows to quantify the metabolic activity of a tumor (glycolysis) and has become a reference tool in oncology for the staging, radiotherapy planning and monitoring tumor response in many cancers (Cacicedo et al., 2016; Fletcher et al., 2008). For primary tumor diagnosis, ${ }^{18} \mathrm{~F}$ FDG-PET imaging showed a significant better sensitivity (93\% vs $65 \%$ ) and specificity (70\% vs $56 \%$ ) over CT (Gambhir et al., 2001). PET imaging allows a more accurate nodal staging of locally advanced head and neck cancer (Kyzas et al., 2008; Yoo et al., 2013), and could result in changing the therapeutic management in nearly $15 \%$ of patients (Lonneux et al., 2010). For patients with cervical node metastases of unknown primary, PET/CT detected a primary tumor in nearly $30 \%$ of patients (Rudmik et al., 2011; Wong et al., 2012; Zhu and Wang, 2013).

Thanks to these potential advantages, PET/CT is recommended for the initial staging and for the treatment decision algorithm of advanced head and neck cancer (Yoo et al., 2013). However, in almost all of these studies, only a visual analysis of PET/CT by physician, based on contrast in uptake between normal tissues and potential tumor (i.e. operator dependent), was performed. Visual analysis was sufficient for diagnosis, staging and detection of recurrence, but with the goal of predicting patient' outcome, quantification is necessary. More recently, quantitative analyses have been introduced in order to overcome some of the limits of the visual methods (Table 1 ). Indeed, quantitative analysis is less operator dependent than visual analysis and can be fully automated, allowing an easier and more objective comparison of the interand intra-patient variations. The main goal of the quantification is to obtain parameters reflecting the tumor activity and/or having a prognostic value.

The aims of this review were to report available evidences on the value of quantitative parameters from PET/CT performed at the diagnosis, during treatment and during follow to predict overalland disease free survival in head and neck cancer and to discuss theirs limits.

\section{Materials and methods}

We performed a systematic electronic search of articles published in PubMed/MEDLINE from January 2000 to march 2016. Our search was restricted to articles reporting data obtained on humans and to English-written articles dealing with locally advanced head and neck cancer and PET/CT. All the articles which did not report data on the prognostic value of PET/CT-related parameters were excluded as well as all the articles which reported data obtained only from visual analyses. Hence this review was focused on the prognostic value of parameters obtained from quantitative or semi-quantitative analyses. We included all the studies reporting data on PET/CT performed before, during or after exclusive RT +/$\mathrm{CT}$, excluding those reporting data from surgical series and/or post-operative radio-chemotherapy. The predictive value of PET at diagnosis, during treatment and during follow up was analyzed separately.

\section{Results}

One hundred and twenty-five studies were identified according to the criteria described above. Seventy-seven studies were excluded since they did not match the inclusion criteria, mainly because they dealt with operated patients (22/77 studies). One retrospective study presenting data on a small population $(<20$ patients) was also excluded. Finally, 45 studies were included in the analysis, for a total of 2928 patients. Table 2 summarizes the main characteristics of the studies included in this analysis, while Table 3 summarizes the principal results of these studies.

\subsection{Predictive value of $18 F D G$ PET before treatment with RT-CT}

Forty-two studies investigated the predictive value of quantitative PET parameters at diagnosis (Table 4). The large majority of these studies analyzed parameters based on Standard Uptake Value (SUV), while only 3 studies performed texture or shape analysis.

\subsubsection{SUVmax and metabolic tumor volume}

Maximum standard uptake value (SUVMax) corresponding to the maximal pixel value in the tumor. Thanks to its ease of use, it was historically the first parameter analyzed. SUVmax was correlated with overall- or disease free survival in 11 studies (Allal et al., 2002; Brun et al., 2002; Castaldi et al., 2012; Chen et al., 2014; Farrag et al., 2010; Higgins et al., 2012; Kitagawa et al., 2003; Machtay et al., 2009; Matoba et al., 2015; Rasmussen et al., 2015; Sanghera et al., 2005). SUVmax allows to identify patients with a high risk of events (death or recurrence). For example, (Rasmussen et al., 2015) analyzed 287 patients with locally advanced head and neck cancers treated with radiotherapy \pm chemotherapy. SUVmax showed a higher predictive value for recurrence than $\mathrm{T}$ stage, $\mathrm{N}$ stage and age. The authors developed a prognostic model of freedom from failure at 2 years, in which including SUVmax significantly increased the predictive value, changing the estimated risk by more than $10 \%$ for $23 \%$ of the patients. In (Allal et al., 2002), 63 patients treated with $\mathrm{RT} \pm \mathrm{CT}$ were prospectively included. Patients presenting a SUVMax $<5.5 \mathrm{~g} / \mathrm{ml}$ had a 3-year DFS of $79 \%$ compared to $42 \%$ for those with SUVmax $>5.5 \mathrm{~g} / \mathrm{ml}(\mathrm{p}=0.005)$. However, the range of cutoff values adopted in published studies to define patients at high or low risk of events markedly varied between 3.7 and $9 \mathrm{~g} / \mathrm{ml}$ (median: 5.8). Noteworthy, also 2 negative studies are available in the literature (Ashamalla et al., 2014; Greven et al., 2001). In (Greven et al., 2001), patients with local recurrence had a mean pretreatment $\mathrm{SUV}_{\mathrm{Max}}$ of $7.7 \mathrm{~g} / \mathrm{ml}$ versus $8.2 \mathrm{~g} / \mathrm{ml}$ for patients without local recurrence. In (Ashamalla et al., 2014), SUVMax was correlated with OS in univariate analysis, but not in multivariate analysis. Only 28 patients were included in this study, which may explain this negative result.

The Metabolic Tumor Volume (MTV), defined as the volume of FDG activity in a tumor assessed by automated volume of interest delineation, and Total Lesion Glycolysis (TLG), defined as MTV x SUVmean, may be more representative of the tumor heterogeneity. The predictive value of MTV was evaluated in 26 studies, with 21 of them also evaluating SUVMax (for a total of 1464 patients). All these studies showed that MTV/TLG were predictive for clinical outcome, with a higher predictive value than SUVmax. In (Chang et al., 2012), 108 patients with nasopharyngeal cancer treated with RT-CT were prospectively included to assess the predictive value of SUVMax, MTV and TLG for DFS and OS. Only Epstein-Barr virus DNA load and TLG of the tumor were significantly correlated with DFS and OS. In particular, patients presenting a TLG value $<65 \mathrm{~g}$ showed a 3 -year DFS of $79.9 \%$ versus $37.4 \%$ for other patients ( $p<0.001$ ), with a hazard ratio of $3.54(p=0.006)$ for DFS and of $4.91(p=0.045)$ for OS.

In two studies, MTV was found to have a higher predictive value than TNM staging (Kao et al., 2012; Romesser et al., 2014). (Romesser et al., 2014) reported data of 100 oropharyngeal cancer, treated with RT-CT (median follow-up: 49 months). MTV at a cutoff of $9.7 \mathrm{ml}$ was correlated with DFS ( $80.3 \%$ vs $56.7 \%, \mathrm{p}=0.015)$ and OS 
Table 1

Most frequently used quantitative parameters in PET imaging.

\begin{tabular}{|c|c|}
\hline Parameters & Definition and method to compute \\
\hline SUV $_{\text {Max }}$ & Maximal pixel value in the tumor \\
\hline SUV Peak & $\begin{array}{l}\text { Average SUV within a small, fixed-size region of interest (ROIpeak) of } 1.2 \mathrm{~cm} \text { diameter, centered on a high-uptake part } \\
\text { of the tumor }\end{array}$ \\
\hline Metabolic Tumor Volume (MTV) & Sum of the volume of voxels with SUV exceeding a certain threshold value in a tumor \\
\hline SUV Mean & Average SUV in the ROI (defined by applying a threshold or by visual assessment) \\
\hline Total Lesion Glycolysis (TLG) & TLG is obtained by multiplying MTV and the mean SUV of the MTV \\
\hline
\end{tabular}

(84.1\% vs $57.8 \% \mathrm{p}=0.008$ ). In multivariate analysis, only MTV was significant while GTV, T stage and N stage did not.

Noteworthy, the reproducibility of the MTV and/or TLG may be limited by the initial definition of these parameters, which is based on a threshold of SUV, absolute (all pixels with SUV value $>x$ ) or relative (all pixels with SUV value $>\mathrm{xx} \%$ of SUVMax). The choice of the threshold for either method may affect the absolute value of the MTV. Six studies compared the predictive value of MTV and/or TLG computed with different thresholds (Cheng et al., 2015; Kao et al., 2012; Lin et al., 2015; Schinagl et al., 2011; Yabuki et al., 2015). In the study by (Schinagl et al., 2011), 4 thresholds (2.5, 40\%, 50\% and adaptive threshold based on liver uptake) were compared for 77 patients treated with RT \pm CT. MTV $40 \%$ was the strongest predictor of DFS and OS. However, even if the predictive value of the other thresholds was slightly lower, they were also correlated with OS and DFS. Same results were reported by the others studies. Based on these results, the use of different thresholds within a reasonable range (between 2 and 3 for an absolute threshold; and between 40 and $50 \%$ for a relative threshold) seems to have no major impact on the predictive value of MTV.

\subsubsection{Texture and shape analysis}

Two different approaches have been used to evaluate tumor heterogeneity, one morphological at macroscopic level (shape of the metabolic area) and the other at pixel level (texture analysis). (Apostolova et al., 2014) used a new parameter to characterize the deviation of the tumor's shape from sphere symmetry (asphericity). The initial assumption of the authors was that "aggressive" tumors are expected to show more irregular shapes, due to necrosis, angiogenesis and extravascular extracellular matrix. In a first study, including patients treated with surgery, radiotherapy or chemotherapy alone, asphericity was correlated with OS and PFS. Based on these results, the authors tried to confirm the predictive value of asphericity in a following study (Hofheinz et al., 2015). Thirty-three patients, with LAHNC treated with RT-CT were included. Using the same cutoff of 20.4 found in (Apostolova et al., 2014), asphericity was correlated with PFS (HR 2.96, p=0.015) and OS (HR 5.9, $\mathrm{p}=0.001$ ).

Two studies evaluated the prognostic value of texture analysis in LAHNC. In the first study (Cheng et al., 2013), including 70 oropharyngeal cancers, TLG and texture uniformity were correlated with OS (HR 5.85 and 0.46 respectively). A 3-point risk scale for DFS and OS was proposed, according to the presence of a uniformity $\leq 0.138$ and a TLG $>122.9 \mathrm{~g}$. One point was given for each factor. Clinical outcome (DFS or OS) was significantly different in the 3 risk groups. These findings were confirmed in an independent series of 88 oropharyngeal cancer patients (Cheng et al., 2015).

\subsection{Predictive value of quantitative PET parameters during chemoradiotherapy}

Early changes in tumor metabolism during radiochemotherapy may be assessed by PET/CT and may be used to tailor treatment. The aims of this adaptive strategy to the treatment' response are to decrease the adverse effect and/or to intensify the treatment, with the final goal to improve the outcome.
Seven studies (374 patients) evaluated the predictive value of PET performed during RT \pm CT (Brun et al., 2002; Castaldi et al., 2012; Chen et al., 2014; Farrag et al., 2010; Hentschel et al., 2011; Min et al., 2016, 2015). All but one of them found a correlation between PET parameters RT \pm CT and clinical outcome. In a study by Min et al., 100 patients received a PET before and 3 weeks after the beginning of treatment (Min et al., 2016). The authors showed that pre-treatment SUVMax and mid-treatment TLG were correlated with 2-year DFS in multivariate analysis ( $83 \%$ vs $71.4 \%, p=0.0019$ and $88.4 \%$ vs $77.2 \%, \mathrm{p}=0.012$, respectively). Moreover, patients presenting pretreatment $\mathrm{TLG}<91 \mathrm{~g}$ and a mid-treatment $\mathrm{TLG}<9.4 \mathrm{~g}$ presented a better 2-year DFS ( $88.1 \%$ vs $61.1 \%, \mathrm{p}=0.001)$ and 2 year OS (90\% vs 67\%, p=0.012). Other parameter,such as SUVMax and MTV were also correlated to DFS and OS, but TLG was the most predictive one. In (Castaldi et al., 2012), which included 24 patients, no predictive value of PET during treatment was shown. However, the decrease of SUVmax between PET at diagnosis and during treatment was highly correlated with 2 -year DFS $(100 \%$ in case of complete response vs 74\% in case of partial response, defined as a reduction of $25 \%$ in tumor 18FDG SUV (Young et al., 1999)).

The optimal time to perform PET during treatment is still unclear. Most of the studies performed the PET before the third week to allow time for adapting therapy. A prospective multicentric study (TEMPORAL) (NCT02469922) is undergoing to assess the predictive value of PET at the 2nd and 4th week of chemoradiotherapy. One hundred twenty-three patients are expected to be included.

\subsection{Predictive value of $18 F D G$ PET after treatment}

After treatment with radiotherapy, PET/CT may be used to identify good responders and avoid useless neck dissection. Twelve studies performed a quantitative or semi quantitative analysis from PET after treatment. All these studies evaluated the SUVMax. A high SUVMax in post treatment was correlated with a poor outcome in 6 studies (Horiuchi et al., 2008; Hoshikawa et al., 2011; Ito et al., 2014; Kim et al., 2016; Kitagawa et al., 2003; Moeller et al., 2010). In (Moeller et al., 2010), 98 patients underwent a PET before and 8 weeks after $\mathrm{RT}+1-\mathrm{CT}$. The authors found that a post-treatment SUVMax $\leq 6 \mathrm{~g} / \mathrm{ml}$ and the variation of SUVMax (in\%) between the pre- and post-irradiation PET/CT were predictive for DFS. In (Kim et al., 2016), a PET was performed 3 months after RT-CT. Seventyeight patients were analyzed. Three-year OS was $87.7 \%$ in patients with SUVMax $<4.4 \mathrm{~g} / \mathrm{ml}$ versus $56.9 \%(\mathrm{p}=0.002)$.

A comparison between visual analysis and quantitative parameters was performed by (Hoshikawa et al., 2009). Thirty-five patients underwent PET before and 5 weeks after RT-CT. Patients with a post-treatment SUVMax value $>3 \mathrm{~g} / \mathrm{ml}$ and decreasing less than $60 \%$ compared to the pre-treatment situation presented a higher risk of recurrence (odds ratio $=61.5, \mathrm{p}<0.0001$ ). The overall accuracy for quantitative analysis was $89.9 \%$ vs $60.9 \%$ for the visual analysis. 
Table 2

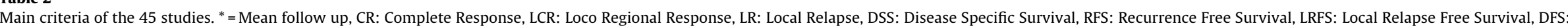
Disease Free Survival, OS: Overall Survival, MTV: Metabolic Tumor Volume, TLG: Total Lesion Glycolysis.

\begin{tabular}{|c|c|c|c|c|c|c|c|c|c|}
\hline Authors & Year & Subject No. & Study Design & $\begin{array}{l}\text { Timing of te } \\
\text { PET-CT }\end{array}$ & Follow up & Localisation & Treatment & End point & $\begin{array}{l}\text { Quantitative PET } \\
\text { parameters }\end{array}$ \\
\hline Greven & 2001 & 45 & Prospective & Pre post & $\mathrm{N} / \mathrm{A}$ & HNC & RT & LR & $\mathrm{SUV}_{\operatorname{Max}}$ \\
\hline Allal & 2002 & 63 & Prospective & Pre & 36 & HNC & $\mathrm{RT} \pm \mathrm{CT}$ & $\begin{array}{l}\text { DFS } \\
\text { OS }\end{array}$ & $\operatorname{SUV}_{\text {Max }}$ \\
\hline Brun & 2002 & 47 & Prospective & Pre and per & 39.6 & HNC & $\mathrm{RT} \pm \mathrm{CT}$ & $\begin{array}{l}\text { CR } \\
\text { LRC } \\
\text { OS }\end{array}$ & $\begin{array}{l}\text { SUV }_{\text {Max }} \\
\text { Metabolic rate FDG }\end{array}$ \\
\hline Kitagawa & 2003 & 20 & Prospective & Pre, post & 52.8 & HNC & CRT & $\mathrm{CR}$ & $\mathrm{SUV}_{\operatorname{Max}}$ \\
\hline Sanghera & 2005 & 12 & Prospective & Pre & 24 & $\mathrm{HNC}$ & RT & OS & $\begin{array}{l}\text { SUV } \\
\text { Sua at } 1 \text { and } 2 \mathrm{~h} \text {, } \\
\text { MUax }_{\text {Mafference }} \text { Dif }\end{array}$ \\
\hline Horiuchi & 2008 & 31 & Retrospective & Pre and post & $\mathrm{N} / \mathrm{A}$ & $\mathrm{HNC}$ & CRT & LR & $\operatorname{SUV}_{\operatorname{Max}}$ \\
\hline Chung & 2009 & 82 & Retrospective & Pre & $34.8^{*}$ & Pharynx & $\mathrm{RT} \pm \mathrm{CT}$ & $\begin{array}{l}\text { LR } \\
\text { DFS } \\
\text { OS }\end{array}$ & $\begin{array}{l}\text { SUV }_{\text {Max }} \\
\text { MTV 2.5 }\end{array}$ \\
\hline La & 2009 & 85 & Retrospective & Pre & $20.4^{*}$ & HNC & $\mathrm{RT} \pm \mathrm{CT}$ & $\begin{array}{l}\text { OS } \\
\text { DSF } \\
\text { LRC }\end{array}$ & $\begin{array}{l}\text { SUV }_{\text {Max }} \\
\text { MTV 50\% }\end{array}$ \\
\hline Machtay & 2009 & 60 & Retrospective & Pre & $\mathrm{N} / \mathrm{A}$ & HNC & $\mathrm{RT} \pm \mathrm{CT}$ & $\begin{array}{l}\text { OS } \\
\text { DFS }\end{array}$ & $\operatorname{SUV}_{\text {Max }}$ \\
\hline Suzuki & 2009 & 45 & Retrospective & Pre & $24^{*}$ & HNC & RT & $\begin{array}{l}\text { OS } \\
\text { DFS }\end{array}$ & $\operatorname{SUV}_{\operatorname{Max}}$ \\
\hline Farrag & 2010 & 43 & Prospective & Pre and Per & 12.7 & $\mathrm{HNC}$ & CRT & OS DFS LRRFS & $\mathrm{SUV}_{\operatorname{Max}}$ \\
\hline Moeller & 2010 & 98 & Prospective & Pre and post & 24 & $\mathrm{HNC}$ & $\mathrm{RT} \pm \mathrm{CT}$ & DFS & $\begin{array}{l}\text { SUV }_{\text {Max }} \mathrm{T} \\
\text { Change in SUV } \\
\text { Max } T\end{array}$ \\
\hline Seol & 2010 & 59 & Retrospective & Pre & $\mathrm{N} / \mathrm{A}$ & HNC & $\mathrm{Neo} C \mathrm{CT} \pm \mathrm{RT}$ & DFS OS & $\begin{array}{l}\text { SUV }_{\text {Max }} \\
\text { SUVMean } \\
\text { MTV } 2.5\end{array}$ \\
\hline Deron & 2011 & 22 & Retrospective & Pre & 20 & $\mathrm{HNC}$ & $\mathrm{RT} \pm \mathrm{CT}$ & DFS OS & $\begin{array}{l}\text { SUV }_{\text {Max }} \\
\text { MTV 50\% }\end{array}$ \\
\hline Hentschel & 2011 & 37 & Prospective & Pre and per & 26 & HNC & CRT & DFS OS LRC & $\begin{array}{l}\text { SUV }_{\text {Max }} \\
\text { SUVMean } \\
\text { MTV 50\% }\end{array}$ \\
\hline Hoshikawa & 2011 & 35 & Prospective & Pre and post & 50 & $\mathrm{HNC}$ & $\mathrm{RT} \pm \mathrm{CT}$ & Recurrence & $\mathrm{SUV}_{\text {Max }}$ \\
\hline Murphy & 2011 & 47 & Retrospective & Post & 34 & $\mathrm{HNC}$ & CRT & DFS OS & $\begin{array}{l}\operatorname{SUV}_{\text {Max }} \\
\text { MTV 2, 2.5, 3, 3.5, } 4 \\
\text { TLG }\end{array}$ \\
\hline
\end{tabular}


Table 2 (Continued)

\begin{tabular}{|c|c|c|c|c|c|c|c|c|c|}
\hline Authors & Year & Subject No. & Study Design & $\begin{array}{l}\text { Timing of te } \\
\text { PET-CT }\end{array}$ & Follow up & Localisation & Treatment & End point & $\begin{array}{l}\text { Quantitative PET } \\
\text { parameters }\end{array}$ \\
\hline Schinagl & 2011 & 77 & Prospective & Pre & 46 & HNC & CRT & DFS OS & $\begin{array}{l}\text { SUV }_{\text {Max }} \\
\text { SUVMean } \\
\text { MTV 40\% } 50 \% \\
\text { MTV 2.5 } \\
\text { GTV - PET }\end{array}$ \\
\hline Castaldi & 2012 & 24 & Prospective & Pre per and post & 29.2 & HNC & CRT & RFS DSS & $\begin{array}{l}\text { SUV }_{\text {Max }} \\
\text { Change in SUVMax } \\
\text { (EORTC criteria) }\end{array}$ \\
\hline Chang & 2012 & 108 & Prospective & Pre & $\mathrm{N} / \mathrm{A}$ & Nasopharynx & CRT & OS DFS LRFS & $\begin{array}{l}\text { SUV }_{\text {Max }} \\
\text { MTV } 2.5 \\
\text { TLG }\end{array}$ \\
\hline Chu & 2012 & 51 & Retrospective & Pre & 17.5 & HNC & $\mathrm{RT} \pm \mathrm{CT}$ & OS DFS & $\begin{array}{l}\text { SUV }_{\text {Max }} \\
\text { MTV50\% } \\
\text { MTV Velocity }\end{array}$ \\
\hline Higgins & 2012 & 88 & Retrospective & Pre & 15 & HNC & $\mathrm{RT} \pm \mathrm{CT}$ & DFS LRC OS & $\begin{array}{l}\text { SUV }_{\text {Max }} \\
\text { SUVMean } \\
\text { TLG (manuelly } \\
\text { delineated) }\end{array}$ \\
\hline Kao & 2012 & 64 & Retrospective & Pre & 24 & Pharynx & $\mathrm{RT} \pm \mathrm{CT}$ & DFS PRFS & MTV $2.53 .040 \% 50 \%$ \\
\hline Romesser & 2012 & 41 & Retrospective & Pre & 24.2 & $\mathrm{HNC}$ & $\mathrm{RT} \pm \mathrm{CT}$ & OS DFS LRFS & $\begin{array}{l}\text { SUV }_{\text {Max }} \\
\text { MTV (Gradient based } \\
\text { method) }\end{array}$ \\
\hline Tang & 2012 & 83 & Retrospective & Pre & 20 & HNC & CRT & $\begin{array}{l}\text { OS } \\
\text { DFS }\end{array}$ & $\begin{array}{l}\text { SUV } \operatorname{Max} \\
\text { MTV 50\% }\end{array}$ \\
\hline Cheng & 2013 & 70 & Retrospective & Pre & $>24$ & Oropharynx & CRT & $\begin{array}{l}\text { OS } \\
\text { DFS }\end{array}$ & $\begin{array}{l}\text { MTV } 2.5 \\
\text { TLG } \\
\text { Textural features }\end{array}$ \\
\hline Ashamalla & 2014 & 28 & Retrospective & Pre and post & $36^{*}$ & HNC & $\mathrm{RT} \pm \mathrm{CT}$ & OS & $\begin{array}{l}\text { SUVMax } \\
\text { SUVMean } \\
\text { Anatomical biologica } \\
\text { value = SUVMax x } \\
\text { greatest tumor } \\
\text { diameter }\end{array}$ \\
\hline Chen & 2014 & 51 & Prospective & Pre and per & 23 & Pharynx & $\mathrm{RT} \pm \mathrm{CT}$ & OS DFS & $\begin{array}{l}\text { SUV }_{\text {Max }} \text { pre and per } \\
\text { SUV reduction ratio }\end{array}$ \\
\hline Hanamoto & 2014 & 118 & Prospective & Pre & $\mathrm{N} / \mathrm{A}$ & HNC & CRT & LR & $\begin{array}{l}\text { SUV }_{\text {Max }} \\
\text { SUVMean } \\
\text { MTV } 2.5 \\
\text { TLG }\end{array}$ \\
\hline Ito & 2014 & 36 & Retrospective & Post & $23.8^{*}$ & HNC & CRT & OS LC & $\mathrm{SUV}_{\mathrm{Max}}$ \\
\hline Romesser & 2014 & 100 & Retrospective & Pre & 49 & Oropharyngeal & CRT & LRC DFS OS & $\begin{array}{l}\text { SUV } \\
\text { MTV } 42 \%\end{array}$ \\
\hline Sager & 2014 & 74 & Retrospective & Pre & 23 & HNC & CRT & DFS OS & $\begin{array}{l}\text { SUV } \operatorname{Max} \\
\text { MTV 50\% }\end{array}$ \\
\hline Akagunduz & 2015 & 62 & Retrospective & Pre & 18 & HNC & $\mathrm{RT} \pm \mathrm{CT}$ & LRFS DFS OS & $\begin{array}{l}\text { SUV }_{\text {Max }} \\
\text { SULMax } \\
\text { MTV (adaptive } \\
\text { threshold based) }\end{array}$ \\
\hline Cheng & 2015 & 88 & Retrospective & Pre & 32 & Oropharynx & CRT & DFS DSS & $\begin{array}{l}\text { MTV } 50 \% 42 \% 2.5 \text { and } \\
\text { adaptive threshold } \\
\text { TLG } \\
\text { Textural features }\end{array}$ \\
\hline
\end{tabular}




\begin{tabular}{|c|c|c|c|c|c|c|c|c|c|}
\hline Hofheinz & 2015 & 37 & Prospective & Pre & $27^{*}$ & HNC & CRT & DFS OS & $\begin{array}{l}\text { SUV }_{\text {Max }} \\
\text { SUVMean } \\
\text { MTV (adaptive } \\
\text { threshold) } \\
\text { TLG } \\
\text { Asphericity }\end{array}$ \\
\hline Lin & 2015 & 91 & Retrospective & Pre & 18 & Pharynx & CRT & $\begin{array}{l}\text { OS } \\
\text { DFS }\end{array}$ & $\begin{array}{l}\text { SUV }_{\text {Max Nodal }} \\
\text { MTV2.5 N } \\
\text { MTV40\% N } \\
\text { MTV50\% N } \\
\text { TLG40\% N } \\
\text { TLG50\% N }\end{array}$ \\
\hline Matoba & 2015 & 33 & Prospective & Pre and post & $\mathrm{N} / \mathrm{A}$ & HNC & CRT & LRC DFS OS & $\begin{array}{l}\text { SUV }_{\text {Max }} \\
\text { EORTC Criteria }\end{array}$ \\
\hline Min & 2015 & 72 & Retrospective & Pre and per & 25 & HNC & CRT & $\begin{array}{l}\text { LRFS } \\
\text { DFS } \\
\text { MFFS } \\
\text { OS }\end{array}$ & $\begin{array}{l}\text { SUV }_{\text {Max }} \\
\text { MTV } 2.5 \\
\text { TLG } \\
\text { Percentage reduction } \\
\text { between per and pre } \\
\text { treatment PET }\end{array}$ \\
\hline Moon & 2015 & 44 & Retrospective & Pre & 34.7 & Nasopharynx & CRT & DFS & $\begin{array}{l}\text { SUV }_{\text {Max }} \\
\text { SUVMean } \\
\text { MTV (adaptive } \\
\text { threshold) } \\
\text { TLG }\end{array}$ \\
\hline Rasmussen & 2015 & 287 & Retrospective & Pre & 32 & HNC & $\mathrm{RT} \pm \mathrm{CT}$ & Time to failure & $\begin{array}{l}\text { SUV }_{\text {Max }} \\
\text { SUVMean } \\
\text { SUVPeak }\end{array}$ \\
\hline Schwartz & 2015 & 74 & Retrospective & Pre and post & 50.4 & HNC & CRT & LR DFS OS & $\begin{array}{l}\text { SUV }_{\text {Max }} \\
\text { SUVPeak } \\
\text { MTV 40\% }\end{array}$ \\
\hline Yabuki & 2015 & 118 & Retrospective & Pre & 36 & Larynx & CRT & $\begin{array}{l}\text { OS } \\
\text { DFS }\end{array}$ & $\begin{array}{l}\text { SUV }_{\text {Max }} \\
\text { MTV 2, 2.5, } 3\end{array}$ \\
\hline Kim & 2016 & 78 & Retrospective & Post & 52.7 & HNC & CRT & DFS OS & $\mathrm{SUV}_{\operatorname{Max}}$ \\
\hline Min & 2016 & 100 & Retrospective & Pre and per & 20 & HNC & $\mathrm{RT} \pm \mathrm{CT}$ & LRFS DFS MFFS OS & $\begin{array}{l}\operatorname{SUV}_{\text {Max }} \\
\text { MTV } 2.5 \\
\text { TLG } \\
\text { Percentage reduction } \\
\text { between per and pre } \\
\text { treatment PET }\end{array}$ \\
\hline
\end{tabular}


Table 3

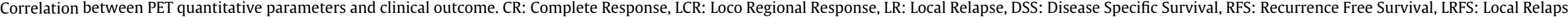
Free Survival, DFS: Disease Free Survival, OS: Overall Survival, MTV: Metabolic Tumor Volume, TLG: Total Lesion Glycolysis.

\begin{tabular}{|c|c|c|c|c|c|c|c|c|}
\hline Timing & Authors & Year & End point & Used PET parameters & $\begin{array}{l}\text { Significant Prognostic } \\
\text { parameters }\end{array}$ & Threshold & Clinical outcome & Hazard Ratio \\
\hline $\begin{array}{l}\text { Pre and } \\
\text { post }\end{array}$ & Greven & 2001 & LR & SUVMax & None & & & \\
\hline Pre & Allal & 2002 & DFS OS & SUVMax & SUVMax (DFS) & 5.5 & 3-year DFS $79 \%$ vs $42 \%$ & $\mathrm{~N} / \mathrm{A}$ \\
\hline Pre and per & Brun & 2002 & $\begin{array}{l}\text { Complete } \\
\text { response (CR) } \\
\text { LRC } \\
\text { OS }\end{array}$ & $\begin{array}{l}\text { SUVmax } \\
\text { Metabolic rate (MR) FDG }\end{array}$ & $\begin{array}{l}\text { Pre Treatment: SUVMax Tumor } \\
\text { (CR and LCR) } \\
\text { Per Treatment: SUVMax Tumor } \\
\text { (CR and LCR), MR Tumor and } \\
\text { lymph node (CR and LCR) } \\
\text { MR FDG per OS }\end{array}$ & $\begin{array}{l}\text { Pre treatment SUVmaxT }=9 \\
\text { Per trt SUVmaxT }=5\end{array}$ & $\begin{array}{l}\text { SUVmax pre trt } \\
\text { CR } 96 \% \text { vs } 64 \%(p=0.01) \\
\text { LRC } 96 \text { vs } 57(p=0.003) \\
\text { MR Tumor per trt } \\
\text { CR } 96 \text { vs } 62 \%(p=0.007) \\
\text { LRC } 96 \text { vs } 55 \%(p=0.002) \\
\text { OS } 72 \% \text { vs } 35 \%(p=0.0042) \\
\text { SUVMax per trt } \\
\text { LCR } 91 \% \text { vs } 62(p=0.031)\end{array}$ & \\
\hline $\begin{array}{l}\text { Pre and } \\
\text { post }\end{array}$ & Kitagawa & 2003 & $\begin{array}{l}\text { Clinical } \\
\text { response }\end{array}$ & SUVMax & SUVMax & $\mathrm{N} / \mathrm{A}$ & $\mathrm{N} / \mathrm{A}$ & $\mathrm{N} / \mathrm{A}$ \\
\hline Pre & Sanghera & 2005 & OS & $\begin{array}{l}\text { SUVMax at } 1 \text { and } 2 \mathrm{~h} \text {, } \\
\text { SUVMax Difference }\end{array}$ & SUV difference & $16 \%$ & $\mathrm{~N} / \mathrm{A}$ & $\mathrm{N} / \mathrm{A}$ \\
\hline $\begin{array}{l}\text { Pre and } \\
\text { post }\end{array}$ & Horiuchi & 2008 & LR & SUVMax & SUVMax Post trt & 3.7 & $\mathrm{~N} / \mathrm{A}$ & \\
\hline Pre & Chung & 2009 & $\begin{array}{l}\text { LR } \\
\text { DFS } \\
\text { OS }\end{array}$ & $\begin{array}{l}\text { SUV Max } \\
\text { MTV } 2.5\end{array}$ & MTV & 40 & & DFS $3.42(p=0.04)$ \\
\hline Pre & La & 2009 & $\begin{array}{l}\text { OS } \\
\text { DFS } \\
\text { LRC }\end{array}$ & $\begin{array}{l}\text { SUVMax } \\
\text { MTV 50\% }\end{array}$ & MTV50\% (OS and DFS) & $\mathrm{N} / \mathrm{A}$ & $\mathrm{N} / \mathrm{A}$ & $\begin{array}{l}\text { Increase of } 17.4 \mathrm{ml} \text { of } \\
\text { MTV50\%= HR } 1.9 \text { (first event) } \\
\text { and } 2.1 \text { (death) }\end{array}$ \\
\hline Pre & Machtay & 2009 & $\begin{array}{l}\text { OS } \\
\text { DFS }\end{array}$ & SUVMax & SUVMax & 9 & $\begin{array}{l}2 \text { year DFS } 76 \text { vs } 37 \%(p=0.007) \\
2 \text { year OS } 82 \% \text { vs } 46 \%(p=0.016)\end{array}$ & $\begin{array}{l}\text { DFS: } 2.41(\mathrm{p}=0.03) \\
\text { OS: } 2.47(\mathrm{p}=0.06)\end{array}$ \\
\hline Pre & Suzuki & 2009 & OS DFS & SUVMax & None & 5.5 & $\mathrm{~N} / \mathrm{A}$ & $\mathrm{N} / \mathrm{A}$ \\
\hline Pre and Per & Farrag & 2010 & OS DFS LRRFS & SUVMax & $\begin{array}{l}\text { Pre: SUVMax (OS) } \\
\text { Per SUVmax (OS) }\end{array}$ & $\begin{array}{l}\text { Pre trt: } 8.11 \\
\text { Per trt: } 4.03\end{array}$ & $\begin{array}{l}\text { 2-year OS } \\
\text { SUVmax pre trt } 81 \% \text { vs } 50 \% \\
(p=0.027) \\
\text { SUVMax Per trt } 82 \text { vs } 47 \% \\
(p=0.026)\end{array}$ & $\mathrm{N} / \mathrm{A}$ \\
\hline $\begin{array}{l}\text { Pre and } \\
\text { post }\end{array}$ & Moeller & 2010 & DFS & $\begin{array}{l}\text { SUVMax T } \\
\text { Change in SUVMax T }\end{array}$ & $\begin{array}{l}\text { SUVMax Post } \\
\text { Change in SUVMax }\end{array}$ & 6 & $\mathrm{~N} / \mathrm{A}$ & $\mathrm{N} / \mathrm{A}$ \\
\hline Pre & Seol & 2010 & DFS OS & $\begin{array}{l}\text { SUVMax } \\
\text { SUVMean } \\
\text { MTV } 2.5\end{array}$ & MTV & $9.3 \mathrm{~cm}^{3}$ & $\mathrm{~N} / \mathrm{A}$ & $\begin{array}{l}\text { DFS: } 2.19(p=0.006) \\
\text { OS: } 1.62(p=0.051)\end{array}$ \\
\hline Pre & Deron & 2011 & DFS OS & $\begin{array}{l}\text { SUVMax } \\
\text { MTV 50\% }\end{array}$ & MTV50(DFS OS) & $31 \mathrm{~cm}^{3}$ & $\mathrm{~N} / \mathrm{A}$ & $\mathrm{N} / \mathrm{A}$ \\
\hline Pre and per & Hentschel & 2011 & DFS OS LRC & $\begin{array}{l}\text { SUVmax } \\
\text { SUVMean } \\
\text { MTV 50\% }\end{array}$ & $\begin{array}{l}\triangle \text { SUVmax10/20 (OS) } \\
\text { MTV50 TEP } 0(\text { OS) }\end{array}$ & $\begin{array}{l}\Delta \text { SUVmax } 10 / 2050 \% \\
\text { MTV50\% } 10.2\end{array}$ & $\begin{array}{l}2 \text { year OS } \\
\Delta \text { SUVmax } 10 / 20 \\
88 \% \text { vs } 38 \%(p=0.02) \\
\text { MTV } 50 \% \\
83 \% \text { vs } 34 \%(p=0.02)\end{array}$ & $\mathrm{N} / \mathrm{A}$ \\
\hline $\begin{array}{l}\text { Pre and } \\
\text { post }\end{array}$ & Hoshikawa & 2011 & Recurrence & SUVMax & $\begin{array}{l}\text { SUVMax Post } \\
\% \text { change in SUV }\end{array}$ & $60 \%$ & $\mathrm{~N} / \mathrm{A}$ & $\begin{array}{l}\text { Odds ratio local control } 61.5 \\
(p<0.001)\end{array}$ \\
\hline Post & Murphy & 2011 & DFS OS & $\begin{array}{l}\text { SUVMax } \\
\text { MTV 2, 2.5, 3, 3.5, } 4 \\
\text { TLG }\end{array}$ & Post trt: MTV2.0 (DFS OS) & $15 \mathrm{~cm}^{3}$ & $\mathrm{~N} / \mathrm{A}$ & $\begin{array}{l}\text { Increase of } 21 \mathrm{~cm}^{3}: 2.5(\mathrm{DFS}) \\
\text { and } 2(\mathrm{OS})\end{array}$ \\
\hline Pre & Schinagl & 2011 & DFS OS & $\begin{array}{l}\text { SUVMax } \\
\text { SUVMean } \\
\text { MTV 40\% } 50 \% \\
\text { MTV } 2.5 \\
\text { GTV - PET }\end{array}$ & $\begin{array}{l}\text { GTV PET (LC DFS OS in oral } \\
\text { cavity and oropharyngeal } \\
\text { cancer) } \\
\text { MTV40\% (DMFS DFS OS) }\end{array}$ & $\mathrm{N} / \mathrm{A}$ & $\mathrm{N} / \mathrm{A}$ & $\mathrm{N} / \mathrm{A}$ \\
\hline
\end{tabular}




\begin{tabular}{|c|c|c|c|c|c|c|c|c|}
\hline $\begin{array}{l}\text { Pre per and } \\
\text { post }\end{array}$ & Castaldi & 2012 & RFS DSS & $\begin{array}{l}\text { SUVMax } \\
\text { Change in SUVMax (EORTC } \\
\text { criteria) }\end{array}$ & EORTC criteria post & $\mathrm{N} / \mathrm{A}$ & $\begin{array}{l}2 \text { year DSS (late TEP) } \\
\text { CR } 100 \% \\
\text { PR } 74 \% \\
\text { PD } 33 \% \\
\text { p }=0.009\end{array}$ & $\mathrm{~N} / \mathrm{A}$ \\
\hline Pre & Chang & 2012 & OS DFS LRFS & $\begin{array}{l}\text { SUVMax } \\
\text { MTV } 2.5 \\
\text { TLG }\end{array}$ & TLG T (OS DFS) & $65 \mathrm{~g}$ & $\begin{array}{l}\text { 3-year DFS } \\
79.9 \% \text { vs } 37.4 \%(\mathrm{p}<0.001)\end{array}$ & $\begin{array}{l}\text { DFS: } 3.54 \\
\text { OS: } 4.9\end{array}$ \\
\hline Pre & Chu & 2012 & OS DFS & $\begin{array}{l}\text { SUVMax } \\
\text { MTV50\% } \\
\text { MTV Velocity (Difference } \\
\text { between the 2 pre } \\
\text { treatment TEP) }\end{array}$ & MTV T & $\mathrm{N} / \mathrm{A}$ & $\mathrm{N} / \mathrm{A}$ & $\begin{array}{l}\text { Increase of } 1 \mathrm{cc} / \text { week }=85 \% \\
\text { increase of the risk of death }\end{array}$ \\
\hline Pre & Higgins & 2012 & DFS LRC OS & $\begin{array}{l}\text { SUVMax } \\
\text { SUVMean } \\
\text { TLG (manuelly delineated) }\end{array}$ & SUVMean (DFS) & 7 (median) & $\begin{array}{l}2 \text { year DFS } \\
82 \% \text { vs } 58 \% \mathrm{p}=0.03\end{array}$ & DFS: $1.14(p=0.014)$ \\
\hline Pre & Као & 2012 & DFS PRFS & MTV $2.53 .040 \% 50 \%$ & MTV 2.5 (DFS PRFS) & $13.6 \mathrm{ml}$ & $\begin{array}{l}\text { 2-year PRFS } \\
72 \% \text { vs } 39 \%(p=0.001) \\
2 \text {-year DFS } \\
68 \% \text { vs } 41 \%(p=0.008)\end{array}$ & $\begin{array}{l}\text { DFS HR } 2.69 p=0.011 \\
\text { PRFS HR } 3.76 p=0.003\end{array}$ \\
\hline Pre & Romesser & 2012 & OS DFS LRFS & $\begin{array}{l}\text { SUVMax } \\
\text { MTV (Gradient based } \\
\text { method) }\end{array}$ & MTV & 7.2 & $\begin{array}{l}2 \text {-year LC } 100 \text { vs } 54.2 \% \\
(p=<0.001) \\
\text { DFS } 94.7 \text { vs } 39.4 \%(p=0.001) \\
\text { OS } 94.7 \text { vs } 64.2(p=0.04)\end{array}$ & $\mathrm{N} / \mathrm{A}$ \\
\hline Pre & Tang & 2012 & OS DFS & $\begin{array}{l}\text { SUVMax } \\
\text { MTV50\% }\end{array}$ & MTV50\% $\mathrm{T}$ (OS and DFS) & $\begin{array}{l}\text { Increase of } 17 \mathrm{~cm}^{3} \\
\text { (differencebetweenfirstandthirdquartiles) }\end{array}$ & $\mathrm{N} / \mathrm{A}$ & $\begin{array}{l}\text { DFS: } 2.07(\mathrm{p}=0.00017) \\
\text { OS: } 1.99(\mathrm{p}=0.0048)\end{array}$ \\
\hline Pre & Cheng & 2013 & PFS DSS OS & $\begin{array}{l}\text { MTV } 2.5 \\
\text { TLG } \\
\text { Normalized gray-level } \\
\text { co-occurence matrix } \\
\text { Neighborhood gray-tone } \\
\text { difference matrix }\end{array}$ & $\begin{array}{l}\text { TLG (PFS DSS OS) } \\
\text { Uniformity (PFS DSS OS) }\end{array}$ & $\begin{array}{l}\text { TLG } 121.9 \mathrm{~g} \\
\text { Uniformity } 4 \text { bins } 0.138\end{array}$ & $\mathrm{~N} / \mathrm{A}$ & $\begin{array}{l}\text { PFS } \\
\text { TLG: } 7.15(p=0.02) \\
\text { Uniformity: } 0.32(p=0.001) \\
\text { OS } \\
\text { TLG: } 5.85(p=0.011) \\
\text { Uniformity: } 0.46(p=0.017)\end{array}$ \\
\hline $\begin{array}{l}\text { Pre and } \\
\text { post }\end{array}$ & Ashamalla & 2014 & OS & $\begin{array}{l}\text { SUVMax } \\
\text { SUVMean } \\
\text { Anatomical biological } \\
\text { value = SUVMax X greatest } \\
\text { tumor diameter }\end{array}$ & None & $\mathrm{N} / \mathrm{A}$ & $\mathrm{N} / \mathrm{A}$ & $\mathrm{N} / \mathrm{A}$ \\
\hline Pre and per & Chen & 2014 & OS DFS & $\begin{array}{l}\text { SUVMax pre et per ( } \mathrm{T} \text { et } \mathrm{N}) \\
\text { SUV reduction ratio }\end{array}$ & SUV reduction ratio tumor & 3.9 & $\begin{array}{l}2 \text {-year DFS } \\
64 \% \text { vs } 41 \%(p=0.045) \\
2 \text { year OS } \\
66 \% \text { vs } 47 \%(p=0.035)\end{array}$ & $\begin{array}{l}\text { DFS: } 2.33 \\
\text { OS: } 2.64\end{array}$ \\
\hline Pre & $\begin{array}{l}\text { Hanamoto } \\
{[67]}\end{array}$ & 2014 & LR & $\begin{array}{l}\text { SUVMax } \\
\text { SUVMean } \\
\text { MTV } 2.5 \\
\text { TLG }\end{array}$ & $\begin{array}{l}\text { For laryngeal and } \\
\text { hypopharyngeal cancer, High } \\
\text { MTV }(>25 \mathrm{ml}) \text { or high TLG } \\
(>144.8 \mathrm{~g})=\text { high risk of partial } \\
\text { response }\end{array}$ & $\mathrm{N} / \mathrm{A}$ & $\mathrm{N} / \mathrm{A}$ & $13.4(p=0.003)$ \\
\hline Post & Ito & 2014 & OS LC & SUVMax & SUVMax (OS) & 6.1 & $\begin{array}{l}\text { OS } 12.1 \text { vs } 44.6 \text { months } \\
(\mathrm{p}<0.001)\end{array}$ & $\mathrm{N} / \mathrm{A}$ \\
\hline Pre & Romesser & 2014 & LRC PFS OS & $\begin{array}{l}\text { SUVMax } \\
\text { MTV } 42 \%\end{array}$ & $\begin{array}{l}\text { MTV (Distant metastasis, } \\
\text { Disease progression or death) }\end{array}$ & 9.7 & $\begin{array}{l}5 \text {-year PFS } \\
80.3 \% \text { vs } 56.7 \%(p=0.015) \\
5 \text {-year OS } \\
84.1 \% \text { vs } 57.9 \%(p=0.008)\end{array}$ & $\begin{array}{l}\text { PFS: HR2.17 } \\
\text { OS: HR } 2.37\end{array}$ \\
\hline Pre & Sager & 2014 & DFS OS & $\begin{array}{l}\text { SUVMax } \\
\text { MTV } 50 \%\end{array}$ & MTV50\% & $\mathrm{N} / \mathrm{A}$ & $\mathrm{N} / \mathrm{A}$ & $\begin{array}{l}\text { DFS: } 2.5 \\
\text { OS: } 2\end{array}$ \\
\hline Pre & Akagunduz & 2015 & LRFS DFS OS & $\begin{array}{l}\text { SUVMax } \\
\text { SULMax } \\
\text { MTV (adaptive threshold } \\
\text { based) }\end{array}$ & $\begin{array}{l}\text { MTV (treatment response, LR, } \\
\text { Disease related death) } \\
\text { SULMax (LR) }\end{array}$ & $\mathrm{N} / \mathrm{A}$ & $\begin{array}{l}3 \text {-year (MTV) } \\
\text { DFS } 75.5 \% \text { vs } 25.3 \% \\
\text { OS } 82.9 \% \text { vs } 55.9 \%\end{array}$ & $\mathrm{~N} / \mathrm{A}$ \\
\hline
\end{tabular}


Table 3 (Continued)

\begin{tabular}{|c|c|c|c|c|c|c|c|c|}
\hline Timing & Authors & Year & End point & Used PET parameters & $\begin{array}{l}\text { Significant Prognostic } \\
\text { parameters }\end{array}$ & Threshold & Clinical outcome & Hazard Ratio \\
\hline Pre & Cheng & 2015 & PFS DSS & $\begin{array}{l}\text { MTV } 50 \% 42 \% 2.5 \text { and } \\
\text { adaptive threshold } \\
\text { TLG } \\
\text { Grey level run lenght } \\
\text { encoding matrix } \\
\text { Grey level size zone matrix }\end{array}$ & $\begin{array}{l}\text { Zone size nonuniformity, } \\
\text { Uniformity, TLG adaptive } \\
\text { threshold (PFS) }\end{array}$ & $\mathrm{N} / \mathrm{A}$ & $\mathrm{N} / \mathrm{A}$ & $\mathrm{N} / \mathrm{A}$ \\
\hline Pre & Hofheinz & 2015 & PFS OS & $\begin{array}{l}\text { SUVMax } \\
\text { SUVMean } \\
\text { MTV (adaptive threshold) } \\
\text { TLG } \\
\text { Aphericity }\end{array}$ & TLG MTV ASP & $\begin{array}{l}\text { MTV } 12.6 \\
\text { TLG } 82.6 \\
\text { ASP } 22 \%\end{array}$ & $\mathrm{~N} / \mathrm{A}$ & $\begin{array}{l}\text { PFS } \\
\text { MTV: } 2.89(\mathrm{p}=0.017) \\
\text { TLG: } 3.11(\mathrm{p}=0.02) \\
\text { ASP: } 3.09(\mathrm{p}=0.015) \\
\text { OS } \\
\text { MTV: } 3.3(\mathrm{p}=0.018) \\
\text { TLG: } 3.32(\mathrm{p}=0.016) \\
\text { ASP: } 5.9(\mathrm{p}=0.001)\end{array}$ \\
\hline Pre & Lin & 2015 & $\begin{array}{l}\text { Nodal relapse } \\
\text { free survival } \\
\text { OS } \\
\text { DFS }\end{array}$ & $\begin{array}{l}\text { SUVMax Nodal } \\
\text { MTV2.5 N } \\
\text { MTV40\% N } \\
\text { MTV50\% N } \\
\text { TLG40\% N } \\
\text { TLG50\% N }\end{array}$ & TLG 40\% (DFS NRFS) & $38 \mathrm{~g}$ & $\mathrm{~N} / \mathrm{A}$ & DFS: $2.12(\mathrm{p}=0.02)$ \\
\hline $\begin{array}{l}\text { Pre and } \\
\text { post }\end{array}$ & Matoba & 2015 & LRC PFS OS & $\begin{array}{l}\text { SUVMax } \\
\text { EORTC Criteria }\end{array}$ & EORTC criteria (OS and PFS) & $\mathrm{N} / \mathrm{A}$ & $\mathrm{N} / \mathrm{A}$ & $\mathrm{N} / \mathrm{A}$ \\
\hline Pre and per & Min & 2015 & $\begin{array}{l}\text { LRFS } \\
\text { DFS } \\
\text { MFFS } \\
\text { OS }\end{array}$ & $\begin{array}{l}\text { SUVMax } \\
\text { MTV } 2.5 \\
\text { TLG } \\
\text { Percentage reduction } \\
\text { between per and pre } \\
\text { treatment PET }\end{array}$ & $\begin{array}{l}\text { SUVMax per trt (DFS) } \\
\text { MTV Pertrt (DFS) } \\
\text { TLG pertrt (LRFS DFS) }\end{array}$ & $\begin{array}{l}\text { SUVMax } 4.25 \\
\text { MTV } 3.3 \\
\text { TLG } 9.4\end{array}$ & $\mathrm{~N} / \mathrm{A}$ & $\mathrm{N} / \mathrm{A}$ \\
\hline Pre & Moon & 2015 & DFS & $\begin{array}{l}\text { SUVMax } \\
\text { SUVMean } \\
\text { MTV (adaptive threshold) } \\
\text { TLG }\end{array}$ & TLG & 7.6 & $\mathrm{~N} / \mathrm{A}$ & DFS: $7.62(\mathrm{p}<0.001)$ \\
\hline Pre & Rasmussen & 2015 & Time to failure & $\begin{array}{l}\text { SUVMax } \\
\text { SUVMean } \\
\text { SUVPeak }\end{array}$ & SUVmax & $\mathrm{N} / \mathrm{A}$ & $\mathrm{N} / \mathrm{A}$ & $\begin{array}{l}\text { Time to failure (for SUVMax } \\
\text { increase from } 25 \text { th to } 75 \text { th } \\
\text { percentile): } 1.34(\mathrm{p}=0.039)\end{array}$ \\
\hline $\begin{array}{l}\text { Pre and } \\
\text { post }\end{array}$ & Schwartz & 2015 & LR PFS OS & $\begin{array}{l}\text { SUVMax } \\
\text { SUVPeak } \\
\text { MTV } 40 \%\end{array}$ & $\begin{array}{l}\text { Primary MTV 40\% (LRR, DM, } \\
\text { DFS) }\end{array}$ & $8.76 \mathrm{~cm}^{3}$ & $\mathrm{~N} / \mathrm{A}$ & $\begin{array}{l}\text { LRR: } 4.01(\mathrm{p}=0.02) \\
\text { PFS: } 2.34(\mathrm{p}=0.05)\end{array}$ \\
\hline Pre & Yabuki & 2015 & DFS OS & MTV 2.53 (for $t$ and $\mathrm{n}$ ) & MTV T 2.5 (OS DFS) & $4.9 \mathrm{ml}$ & $\begin{array}{l}3 \text {-year DFS } 92.9 \% \text { vs } 38.6 \% \\
\text { ( }<0.001 \text { ) } \\
3 \text {-year OS } 95.35 \% \text { vs } 59.27 \% \\
(p<0.001)\end{array}$ & $\begin{array}{l}\text { DFS: } 6.97(p=0.001) \\
\text { OS: } 1.96(p=0.002)\end{array}$ \\
\hline Post & Kim & 2016 & PFS OS & SUVMax & SUVMax (DFS and OS) & 4.4 & $\begin{array}{l}3 \text {-year PFS } 81.1 \% \text { vs } 42.9 \% \\
3 \text {-year OS } 87.7 \% \text { vs } 56.9 \%\end{array}$ & $\begin{array}{l}\text { PFS: } 4.79(\mathrm{p}<0.001) \\
\text { OS: } 4.25(\mathrm{p}=0.005)\end{array}$ \\
\hline Pre and per & Min & 2016 & $\begin{array}{l}\text { LRFS DFS MFFS } \\
\text { OS }\end{array}$ & $\begin{array}{l}\text { SUVMax } \\
\text { MTV } 2.5 \\
\text { TLG } \\
\text { Percentage reduction } \\
\text { between per and pre } \\
\text { treatment PET }\end{array}$ & $\begin{array}{l}\text { TLG pertrt (DFS) } \\
\text { SUVMax per (DFS MFFS) } \\
\text { MTV per (DFS OS) } \\
\text { TLG per (DFS) }\end{array}$ & $\begin{array}{l}\text { TLG per trt } 9.4 \\
\text { SUVMax pre } 11.45 \text { and per } 4.25 \\
\text { MTV pre } 21.95 \text { and per } 3.3 \\
\text { TLG pre } 91.75 \text { and per } 9.4\end{array}$ & $\begin{array}{l}\text { TLG } \\
2 \text {-year DFS } 85.9 \% \text { vs } 60.8 \% \\
(p=0.005) \\
\text { MTV } \\
2 \text {-year DFS } 83.2 \% \text { vs } 62.3 \% \\
\text { ( } p=0.018) \\
\text { SUVMax } \\
\text { 2-year DFS } 82 \% \text { vs } 64.5 \% \\
(p=0.025)\end{array}$ & $\begin{array}{l}\text { TLG DFS: } 7.7 \\
\text { MTV DFS: } 4.29 \\
\text { SUVMax: } 4.18\end{array}$ \\
\hline
\end{tabular}


Table 4

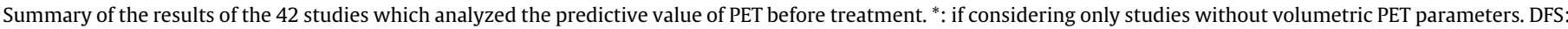
Disease Free Survival, OS: Overall Survival, MTV: Metabolic Tumor Volume, TLG: Total Lesion Glycolysis.

\begin{tabular}{|c|c|c|c|c|}
\hline $\begin{array}{l}\text { Quantitative } \\
\text { parameters }\end{array}$ & $\begin{array}{l}\text { Correlation with } \\
\text { DFS/OS }\end{array}$ & $\begin{array}{l}\text { Number of positive } \\
\text { studies/total studies }\end{array}$ & Strength & Weakness \\
\hline SUV $_{\text {Max }}$ & Poorly & $14 / 38\left(11 / 14^{*}\right)$ & Ease of use & $\begin{array}{l}\text { Poorly reproducible } \\
\text { No data concerning heterogeneity }\end{array}$ \\
\hline SUV $_{\text {Peak }}$ & $?$ & $0 / 2$ & More robust than $\mathrm{SUV}_{\mathrm{Max}}$ & $\begin{array}{l}\text { May not be representative of } \\
\text { nonhomogeneous overall tumor } \\
\text { uptake } \\
\text { Ideal size of the ROI is still unclear }\end{array}$ \\
\hline SUV $_{\text {Mean }}$ & No & $1 / 9$ & - & - \\
\hline MTV/TLG & Yes & $26 / 26$ & $\begin{array}{l}\text { Represent the heterogeneity of the } \\
\text { tumor uptake } \\
\text { Ease of use }\end{array}$ & $\begin{array}{l}\text { No clearly segmentation method } \\
\text { No data concerning spatial } \\
\text { relationships }\end{array}$ \\
\hline $\begin{array}{l}\text { Shape/ } \\
\text { Texture analysis }\end{array}$ & ? & $3 / 3$ & $\begin{array}{l}\text { Represent the heterogeneity of the } \\
\text { tumor }\end{array}$ & $\begin{array}{l}\text { No standardized method/Experimental } \\
\text { Which correlation with histology? }\end{array}$ \\
\hline
\end{tabular}


Fig. 1. Heterogeneity measures do not characterize the spatial relationships between voxels. The two tumors in the upper row have identical SUV histograms, although their visual aspect is very dissimilar.

\section{Discussion and conclusion}

This overview of the available literature shows that MTV and TLG are well correlated with clinical outcome (Local control, Disease Free Survival and overall survical). Most of the available data deal with the intensity of the metabolism, calculated from the SUV, a quantitative parameter used to normalize the uptake of ${ }^{18} \mathrm{~F}$ FDG. In practice, SUV is defined as a ratio of tissue radioactivity concentration and the injected dose adjusted by body weight (SUV $\mathrm{bw}_{\mathrm{b}}$ with BW for body weight). Intensity of the metabolism can be analyzed using histogram-based method, which represents the voxel value frequency distribution. This method includes in particular the four histogram moments, i.e., the mean (corresponding to SUVMean), the maximum (corresponding to SUVMax), the median, the skewness (asymmetry of the histogram) and kurtosis (degree of peakedness of a distribution). However, it did not take into account the spatial relationship between voxel values (Fig. 1).

The maximum SUV (SUVMax) corresponds to the maximal pixel value in the tumor. Thanks to its ease of use, it is one of the most used parameters in the clinical practice. However, this value is highly dependent from noise, duration and parameters of acquisition, and so is considered to be poorly reproducible (Boellaard et al., 2004; Nahmias and Wahl, 2008; Nakamoto et al., 2002). This point may explain the wide range of cut-off value for SUVmax reported in the available studies (from 3.7 to $9 \mathrm{~g} / \mathrm{ml}$ ), limiting the generalization of the use of SUVmax for the whole population. Peak SUV $\left(S_{U V} V_{\text {Peak }}\right)$, defined as the average SUV within a small region of interest $(1.2 \mathrm{~cm}$ of diameter) around the $\mathrm{SUV}_{\mathrm{Max}}$, is a more robust alternative to $S_{\text {SUax }}$. However, SUV $\mathrm{V}_{\text {Peak }}$ may not be representative of nonhomogeneous overall tumor uptake, and the ideal size of the ROI is still unclear (Lee et al., 2007).

Others volumetric parameters like the MTV, the mean SUV within the tumor volume or the TLG are used to represent the heterogeneity of the tumor uptake. The predictive value for clinical outcome of these parameters seems to be higher than $S_{U V} V_{M a x}$. However, uptake in PET may be due to inflammatory or infectious reaction. Furthermore, the physiological uptake surrounding organs can also be a source of loss of specificity in the analysis of the signal. A major difficulty in the analysis of PET is to differentiate the tumor signal from the non-tumor signal. PET imaging suffers from a low contrast and spatial resolution, with a high noise background and partial effect volume. Tumor delineation may change depending on the chosen segmentation method. One of the most used automatic method is to use a threshold, between 2 and 3 (absolute value) or $40-50 \%$ (relative value of SUVmax).

One important issue concerning the predictive value of MTV is the lack of external validation. Most of the studies were monocentric, using the same PET/CT for all patients. Only two studies performed a validation on an independent dataset (Hofheinz et al., 2015; Tang et al., 2012). The first study (La et al., 2009) included 85 patients and showed that an increase of MTV of $17 \mathrm{~cm}^{3}$ (from the 25th to 75th percentile) was significantly correlated with an increased risk of death (HR 2.1). The authors validated their results on a dataset of 83 patients treated in the same institution after the original dataset (Tang et al., 2012). Based on (Apostolova et al., 2014), (Hofheinz et al., 2015) used a cutoff of TLG of $58.7 \mathrm{ml}$. They showed a correlation of TLG only with better DFS (HR 3.01, $\mathrm{p}=0.048$ ) but not with better OS (HR 2.02, $\mathrm{p}=0.22$ ). After adjusting the cutoff at a value of $141 \mathrm{ml}$, TLG was also correlated with OS (HR 3.32, p = 0.016). Such methodologies and findings highlight the difficulty in identifying a cutoff which may be tested on external dataset of patients. The use of international guidelines, like the European Association of Nuclear Medecine guidelines for tumor imaging (Boellaard et al., 2015), by harmonizing quantitative FDG PET/CT imaging procedures in multicentre studies and quantitative interpretation criteria, may increases the reproducibility of PET studies.

The spatial relationships between the voxel values within the tumor may be assessed by texture analyses. Texture analyses aim to characterize the internal metabolism morphology of the tumors. From a technical point of view, they characterize the transitions between voxel values. Several approaches exist and they all rely on a quantification of spatial scales organization and directions in images. Most approaches are computing the latter in two dimensions on a slice basis. The most widely used method is the Gray Level Co-occurrence Matrix and consists in calculating matrices count- 


\section{Unresolved questions and controversies}
1. Reproducibility of PET parameters between different machines and/or centre
2. Which methods (Manually, relative, absolute or adaptive segmentation) and which threshold to compute MTV?
3. When should perform PET during radiotherapy?
4. Which methods for texture and shape analysis?

ing the co-occurrences of two voxels values separated from a set of fixed distances and along set of fixed directions. Several statistics can be computed on these matrices to quantify textural properties (e.g., correlation, contrast, energy). Another popular approach is to apply image filters with various scales and directional properties to continuously quantify transitions between image voxels. One popular example is the isotropic Mexican hat filter (also called Laplacian of Gaussian filter). A comprehensive review of methods for 3-D texture analysis is available in (Depeursinge et al., 2014). This kind of analysis seems promising, but its use should still be considered experimental and limited to clinical studies.

\section{Conflicts of interest}

None.

\section{Acknowledgment}

The authors wish to thank Ms S.Colombe who assisted in proofreading the manuscript.

\section{References}

Allal, A.S., Dulguerov, P., Allaoua, M., Haenggeli, C.A., El-Ghazi el, A., Lehmann, W., Slosman, D.O., 2002. Standardized uptake value of 2-[(18)F]

fluoro-2-deoxy-D-glucose in predicting outcome in head and neck carcinomas treated by radiotherapy with or without chemotherapy. J. Clin. Oncol. 20 (5), 1398-1404.

Apostolova, I., Steffen, I.G., Wedel, F., Lougovski, A., Marnitz, S., Derlin, T., Amthauer, H., Buchert, R., Hofheinz, F., Brenner, W., 2014. Asphericity of pretherapeutic tumour FDG uptake provides independent prognostic value in head-and-neck cancer. Eur. Radiol. 24 (9), 2077-2087.

Ashamalla, H., Mattes, M., Guirguis, A., Zaidi, A., Mokhtar, B., Tejwani, A., 2014. The anatomical biological value on pretreatment (18)F-fluorodeoxyglucose positron emission tomography computed tomography predicts response and survival in locally advanced head and neck cancer. World J. Nucl. Med. 13 (2), 102-107.

Boellaard, R., Krak, N.C., Hoekstra, O.S., Lammertsma, A.A., 2004. Effects of noise, image resolution, and ROI definition on the accuracy of standard uptake values: a simulation study. J. Nucl. Med. 45 (9), 1519-1527.

Boellaard, R., Delgado-Bolton, R., Oyen, W.J., Giammarile, F., Tatsch, K., Eschner, W., Verzijlbergen, F.J., Barrington, S.F., Pike, L.C., Weber, W.A., Stroobants, S. Delbeke, D., Donohoe, K.J., Holbrook, S., Graham, M.M., Testanera, G., Hoekstra, O.S., Zijlstra, J., Visser, E., Hoekstra, C.J., Pruim, J., Willemsen, A., Arends, B. Kotzerke, J., Bockisch, A., Beyer, T., Chiti, A., Krause, B.J., European Association of Nuclear, M., 2015. FDG PET/CT: EANM procedure guidelines for tumour imaging: version 2.0. Eur. J. Nucl. Med. Mol. Imaging 42 (2), 328-354.

Brun, E., Kjellen, E., Tennvall, J., Ohlsson, T., Sandell, A., Perfekt, R., Perfekt, R., Wennerberg, J., Strand, S.E., 2002. FDG PET studies during treatment: prediction of therapy outcome in head and neck squamous cell carcinoma. Head Neck 24 (2), 127-135.

Cacicedo, J., Navarro, A., Del Hoyo, O., Gomez-Iturriaga, A., Alongi, F., Medina, J.A., Elicin, O., Skanjeti, A., Giammarile, F., Bilbao, P., Casquero, F., de Bari, B., Dal Pra, A., 2016. Role of fluorine-18 fluorodeoxyglucose PET/CT in head and neck oncology: the point of view of the radiation oncologist. Br. J. Radiol., 20160217.

Castaldi, P., Rufini, V., Bussu, F., Micciche, F., Dinapoli, N., Autorino, R., Lago, M., De Corso, E., Almadori, G., Galli, J., Paludetti, G., Giordano, A., Valentini, V., 2012. Can early and late18F-FDG PET-CT be used as prognostic factors for the clinical outcome of patients with locally advanced head and neck cancer treated with radio-chemotherapy? Radiother. Oncol.: J. Eur. Soc. Ther. Radiol. Oncol. 103 (1), 63-68.

Chajon, E., Lafond, C., Louvel, G., Castelli, J., Williaume, D., Henry, O., Jegoux, F., Vauleon, E., Manens, J.P., Le Prise, E., de Crevoisier, R., 2013. Salivary gland-sparing other than parotid-sparing in definitive head-and-neck intensity-modulated radiotherapy does not seem to jeopardize local control Radiat. Oncol. 8, 132.

Chang, K.P., Tsang, N.M., Liao, C.T., Hsu, C.L., Chung, M.J., Lo, C.W., Chan, S.C., Ng, S.H., Wang, H.M., Yen, T.C., 2012. Prognostic significance of 18F-FDG PET parameters and plasma Epstein-Barr virus DNA load in patients with nasopharyngeal carcinoma. J. Nucl. Med. 53 (1), 21-28.

Chen, S.W., Hsieh, T.C., Yen, K.Y., Yang, S.N., Wang, Y.C., Chien, C.R., Liang, J.A., Kao, C.H., 2014. Interim FDG PET/CT for predicting the outcome in patients with head and neck cancer. Laryngoscope 124 (12), 2732-2738.

Cheng, N.M., Fang, Y.H., Chang, J.T., Huang, C.G., Tsan, D.L., Ng, S.H., Wang, H.M., Lin, C.Y., Liao, C.T., Yen, T.C., 2013. Textural features of pretreatment 18F-FDG PET/CT images: prognostic significance in patients with advanced T-stage oropharyngeal squamous cell carcinoma. J. Nucl. Med. 54 (10), 1703-1709.

Cheng, N.M., Fang, Y.H., Lee, L.Y., Chang, J.T., Tsan, D.L., Ng, S.H., Wang, H.M., Liao, C.T., Yang, L.Y., Hsu, C.H., Yen, T.C., 2015. Zone-size nonuniformity of 18F-FDG PET regional textural features predicts survival in patients with oropharyngeal cancer. Eur. J. Nucl. Med. Mol. Imaging 42 (3), 419-428.

Depeursinge, A., Foncubierta-Rodriguez, A., Van De Ville, D., Muller, H., 2014. Three-dimensional solid texture analysis in biomedical imaging: review and opportunities. Med. Image Anal. 18 (1), 176-196.

Edge, S.B., Compton, C.C., 2010. The American Joint Committee on Cancer: the 7th edition of the AJCC cancer staging manual and the future of TNM. Ann. Surg. Oncol. 17 (6), 1471-1474.

Farrag, A., Ceulemans, G., Voordeckers, M., Everaert, H., Storme, G., 2010. Can 18 F-FDG-PET response during radiotherapy be used as a predictive factor for the outcome of head and neck cancer patients? Nucl. Med. Commun. 31 (6), 495-501.

Fletcher, J.W., Djulbegovic, B., Soares, H.P., Siegel, B.A., Lowe, V.J., Lyman, G.H., Coleman, R.E., Wahl, R., Paschold, J.C., Avril, N., Einhorn, L.H., Suh, W.W., Samson, D., Delbeke, D., Gorman, M., Shields, A.F., 2008. Recommendations on the use of 18F-FDG PET in oncology. J. Nucl. Med. 49 (3), 480-508.

Gambhir, S.S., Czernin, J., Schwimmer, J., Silverman, D.H., Coleman, R.E., Phelps, M.E., 2001. A tabulated summary of the FDG PET literature. J. Nucl. Med. 42 (Suppl. 5), 1S-93S.

Greven, K.M., Williams 3rd, D.W., McGuirt Sr., W.F., Harkness, B.A., D’Agostino Jr., R.B., Keyes Jr., J.W., Watson Jr., N.E., 2001. Serial positron emission tomography scans following radiation therapy of patients with head and neck cancer. Head Neck 23 (11), 942-946.

Hentschel, M., Appold, S., Schreiber, A., Abolmaali, N., Abramyuk, A., Dorr, W., Kotzerke, J., Baumann, M., Zophel, K., 2011. Early FDG PET at 10 or 20 Gy under chemoradiotherapy is prognostic for locoregional control and overall survival in patients with head and neck cancer. Eur. J. Nucl. Med. Mol. Imaging 38 (7), 1203-1211.

Higgins, K.A., Hoang, J.K., Roach, M.C., Chino, J., Yoo, D.S., Turkington, T.G., Brizel, D.M., 2012. Analysis of pretreatment FDG-PET SUV parameters in head-and-neck cancer: tumor SUVmean has superior prognostic value. Int. J. Radiat. Oncol. Biol. Phys. 82 (2), 548-553.

Hofheinz, F., Lougovski, A., Zophel, K., Hentschel, M., Steffen, I.G., Apostolova, I., Wedel, F., Buchert, R., Baumann, M., Brenner, W., Kotzerke, J., van den Hoff, J., 2015. Increased evidence for the prognostic value of primary tumor asphericity in pretherapeutic FDG PET for risk stratification in patients with head and neck cancer. Eur. J. Nucl. Med. Mol. Imaging 42 (3), 429-437.

Horiuchi, C., Taguchi, T., Yoshida, T., Nishimura, G., Kawakami, M., Tanigaki, Y., Matsuda, H., Mikami, Y., Oka, T., Inoue, T., Tsukuda, M., 2008. Early assessment of clinical response to concurrent chemoradiotherapy in head and neck carcinoma using fluoro-2-deoxy-d-glucose positron emission tomography. Auris Nasus Larynx 35 (1), 103-108.

Hoshikawa, H., Mitani, T., Nishiyama, Y., Yamamoto, Y., Ohkawa, M., Mori, N., 2009 Evaluation of the therapeutic effects and recurrence for head and neck cancer after chemoradiotherapy by FDG-PET. Auris Nasus Larynx 36 (2), 192-198.

Hoshikawa, H., Kishino, T., Nishiyama, Y., Yamamoto, Y., Yonezaki, M., Mori, N., 2011. Early prediction of local control in head and neck cancer after chemoradiotherapy by FDG-PET. Nucl. Med. Commun. 32 (8), 684-689.

Ito, K., Shimoji, K., Miyata, Y., Kamiya, K., Minamimoto, R., Kubota, K., Okasaki, M., Morooka, M., Yokoyama, J., 2014. Prognostic value of post-treatment (18)F-FDG PET/CT for advanced head and neck cancer after combined intra-arterial chemotherapy and radiotherapy. Chin. J. Cancer. Res. 26 (1), 30-37.

Kao, C.H., Lin, S.C., Hsieh, T.C., Yen, K.Y., Yang, S.N., Wang, Y.C., Liang, J.A., Hua, C.H., Chen, S.W., 2012. Use of pretreatment metabolic tumour volumes to predict the outcome of pharyngeal cancer treated by definitive radiotherapy. Eur. J. Nucl. Med. Mol. Imaging 39 (8), 1297-1305.

Kim, R., Ock, C.Y., Keam, B., Kim, T.M., Kim, J.H., Paeng, J.C., Kwon, S.K., Hah, J.H., Kwon, T.K., Kim, D.W., Wu, H.G., Sung, M.W., Heo, D.S., 2016. Predictive and prognostic value of PET/CT imaging post-chemoradiotherapy and clinical decision-making consequences in locally advanced head \& neck squamous cell carcinoma: a retrospective study. BMC Cancer 16 (1), 116.

Kitagawa, Y., Sano, K., Nishizawa, S., Nakamura, M., Ogasawara, T., Sadato, N. Yonekura, Y., 2003. FDG-PET for prediction of tumour aggressiveness and response to intra-arterial chemotherapy and radiotherapy in head and neck cancer. Eur. J. Nucl. Med. Mol. Imaging 30 (1), 63-71.

Kyzas, P.A., Evangelou, E., Denaxa-Kyza, D., Ioannidis, J.P., 2008. 18F-fluorodeoxyglucose positron emission tomography to evaluate cervical node metastases in patients with head and neck squamous cell carcinoma: a meta-analysis. J. Natl. Cancer Inst. 100 (10), 712-720.

La, T.H., Filion, E.J., Turnbull, B.B., Chu, J.N., Lee, P., Nguyen, K., Maxim, P., Quon, A., Graves, E.E., Loo Jr., B.W., Le, Q.T., 2009. Metabolic tumor volume predicts for 
recurrence and death in head-and-neck cancer. Int. J. Radiat. Oncol. Biol. Phys. 74 (5), 1335-1341.

Lee, P., Weerasuriya, D.K., Lavori, P.W., Quon, A., Hara, W., Maxim, P.G., Le, Q.T., Wakelee, H.A., Donington, J.S., Graves, E.E., Loo Jr., B.W., 2007. Metabolic tumor burden predicts for disease progression and death in lung cancer. Int. J. Radiat. Oncol. Biol. Phys. 69 (2), 328-333.

Lin, Y.C., Chen, S.W., Hsieh, T.C., Yen, K.Y., Yang, S.N., Wang, Y.C., Kao, C.H., 2015 Risk stratification of metastatic neck nodes by $\mathrm{CT}$ and PET in patients with head and neck cancer receiving definitive radiotherapy. J. Nucl. Med. 56 (2), 183-189.

Lonneux, M., Hamoir, M., Reychler, H., Maingon, P., Duvillard, C., Calais, G., Bridji, B., Digue, L., Toubeau, M., Gregoire, V., 2010. Positron emission tomography with [18F]fluorodeoxyglucose improves staging and patient management in patients with head and neck squamous cell carcinoma: a multicenter prospective study. J. Clin. Oncol. 28 (7), 1190-1195.

Machtay, M., Natwa, M., Andrel, J., Hyslop, T., Anne, P.R., Lavarino, J., Intenzo, C.M., Keane, W., 2009. Pretreatment FDG-PET standardized uptake value as a prognostic factor for outcome in head and neck cancer. Head Neck 31 (2), 195-201.

Matoba, M., Tuji, H., Shimode, Y., Kondo, T., Oota, K., Tonami, H., 2015. Lesion regression rate based on RECIST: prediction of treatment outcome in patients with head and neck cancer treated with chemoradiotherapy compared with FDG PET-CT. J. Radiat. Res. 56 (3), 553-560.

Min, M., Lin, P., Lee, M.T., Shon, I.H., Lin, M., Forstner, D., Bray, V., Chicco, A., Tieu, M.T., Fowler, A., 2015. Prognostic role of metabolic parameters of (18)F-FDC PET-CT scan performed during radiation therapy in locally advanced head and neck squamous cell carcinoma. Eur. J. Nucl. Med. Mol. Imaging 42 (13), 1984-1994.

Min, M., Lin, P., Lee, M., Shon, I.H., Lin, M., Forstner, D., Tieu, M.T., Chicco, A., Bray, V., Fowler, A., 2016. 18F-FDG PET-CT performed before and during radiation therapy of head and neck squamous cell carcinoma: are they independent or complementary to each other? J. Med. Imaging. Radiat. Oncol, 60 (3), 433-440.

Moeller, B.J., Rana, V., Cannon, B.A., Williams, M.D., Sturgis, E.M., Ginsberg, L.E., Macapinlac, H.A., Lee, J.J., Ang, K.K., Chao, K.S., Chronowski, G.M., Frank, S.J., Morrison, W.H., Rosenthal, D.I., Weber, R.S., Garden, A.S., Lippman, S.M., Schwartz, D.L., 2010. Prospective imaging assessment of mortality risk after head-and-neck radiotherapy. Int. J. Radiat. Oncol. Biol. Phys. 78 (3), 667-674

Murphy, J.D., La, T.H., Chu, K., Quon, A., Fischbein, N.J., Maxim, P.G., Graves, E.E., Loo Jr., B.W., Le, Q.T., 2011. Postradiation metabolic tumor volume predicts outcome in head-and-neck cancer. Int. J. Radiat. Oncol. Biol. Phys. 80 (2), 514-521.

Nahmias, C., Wahl, L.M., 2008. Reproducibility of standardized uptake value measurements determined by 18F-FDG PET in malignant tumors. J. Nucl. Med. 49 (11), 1804-1808.

Nakamoto, Y., Zasadny, K.R., Minn, H., Wahl, R.L., 2002. Reproducibility of common semi-quantitative parameters for evaluating lung cancer glucose metabolism with positron emission tomography using 2-deoxy-2-[18F]fluoro-D-glucose. Mol. Imaging Biol. 4 (2), 171-178.

Parkin, D.M., Bray, F., Ferlay, J., Pisani, P., 2005. Global cancer statistics, 2002. CA Cancer J. Clin. 55 (2), 74-108.

Pignon, J.P., Bourhis, J., Domenge, C., Designe, L., 2000. Chemotherapy added to locoregional treatment for head and neck squamous-cell carcinoma: three meta-analyses of updated individual data. MACH-NC Collaborative Group. Meta-Analysis of Chemotherapy on Head and Neck Cancer. Lancet 355 (9208), 949-955.
Rasmussen, J.H., Vogelius, I.R., Fischer, B.M., Friborg, J., Aznar, M.C., Persson, G.F., Hakansson, K., Kristensen, C.A., Bentzen, S.M., Specht, L., 2015. Prognostic value of $18 \mathrm{~F}$-fludeoxyglucose uptake in 287 patients with head and neck squamous cell carcinoma. Head Neck 37 (9), 1274-1281.

Romesser, P.B., Lim, R., Spratt, D.E., Setton, J., Riaz, N., Lok, B., Rao, S., Sherman, E.J., Schoder, H., Lee, N.Y., 2014. The relative prognostic utility of standardized uptake value, gross tumor volume, and metabolic tumor volume in oropharyngeal cancer patients treated with platinum based concurrent chemoradiation with a pre-treatment [(18)F] fluorodeoxyglucose positron emission tomography scan. Oral Oncol. 50 (9), 802-808.

Rudmik, L., Lau, H.Y., Matthews, T.W., Bosch, J.D., Kloiber, R., Molnar, C.P., Dort, J.C. 2011. Clinical utility of PET/CT in the evaluation of head and neck squamous cell carcinoma with an unknown primary: a prospective clinical trial. Head Neck 33 (7), 935-940.

Sanghera, B., Wong, W.L., Lodge, M.A., Hain, S., Stott, D., Lowe, J., Lemon, C., Goodchild, K., Saunders, M., 2005. Potential novel application of dual time point SUV measurements as a predictor of survival in head and neck cancer. Nucl. Med. Commun. 26 (10), 861-867.

Schinagl, D.A., Span, P.N., Oyen, W.J., Kaanders, J.H., 2011. Can FDG PET predict radiation treatment outcome in head and neck cancer? Results of a prospective study. Eur. J. Nucl. Med. Mol. Imaging 38 (8), 1449-1458.

Schwartz, D.L., Harris, J., Yao, M., Rosenthal, D.I., Opanowski, A., Levering, A., Ang, K.K., Trotti, A.M., Garden, A.S., Jones, C.U., Harari, P., Foote, R., Holland, J., Zhang, Q., Le, Q.T., 2015. Metabolic tumor volume as a prognostic imaging-based biomarker for head-and-neck cancer: pilot results from Radiation Therapy Oncology Group protocol 0522. Int. J. Radiat. Oncol. Biol. Phys. 91 (4), 721-729.

St Guily, J.L., Borget, I., Vainchtock, A., Remy, V., Takizawa, C., 2010. Head and neck cancers in France: an analysis of the hospital medical information system (PMSI) database. Head Neck Oncol. 2, 22.

Tang, C., Murphy, J.D., Khong, B., La, T.H., Kong, C., Fischbein, N.J., Colevas, A.D., Iagaru, A.H., Graves, E.E., Loo Jr., B.W., Le, Q.T., 2012. Validation that metabolic tumor volume predicts outcome in head-and-neck cancer. Int. J. Radiat. Oncol. Biol. Phys. 83 (5), 1514-1520.

Wong, W.L., Sonoda, L.I., Gharpurhy, A., Gollub, F., Wellsted, D., Goodchild, K., Lemon, C., Farrell, R., Saunders, M., 2012. 18F-fluorodeoxyglucose positron emission tomography/computed tomography in the assessment of occult primary head and neck cancers?an audit and review of published studies. Clin. Oncol. (R Coll Radiol) 24 (3), 190-195.

Yabuki, K., Shiono, O., Komatsu, M., Sano, D., Nishimura, G., Takahashi, M., Taguchi, T., Inoue, T., Oridate, N., 2015. Predictive and prognostic value of metabolic tumor volume (MTV) in patients with laryngeal carcinoma treated by radiotherapy (RT)/concurrent chemoradiotherapy (CCRT). PLoS One 10 (2), e0117924.

Yoo, J., Henderson, S., Walker-Dilks, C., 2013. Evidence-based guideline recommendations on the use of positron emission tomography imaging in head and neck cancer. Clin. Oncol. (R Coll Radiol) 25 (4), e33-66.

Young, H., Baum, R., Cremerius, U., Herholz, K., Hoekstra, O., Lammertsma, A.A., Pruim, J., Price, P., 1999. Measurement of clinical and subclinical tumour response using [18F]-fluorodeoxyglucose and positron emission tomography: review and 1999 EORTC recommendations. Eur. J. Cancer 35 (13), 1773-1782.

Zhu, L., Wang, N., 2013. 18F-fluorodeoxyglucose positron emission tomography-computed tomography as a diagnostic tool in patients with cervical nodal metastases of unknown primary site: a meta-analysis. Surg. Oncol. 22 (3), 190-194. 